\title{
Correlative analysis of shoe insoles acting in moist and cold/warm environment
}

\author{
Barbu Braun ${ }^{1, *}$, and Mihaela Baritz ${ }^{1}$ \\ ${ }^{1}$ Transilvania University of Brasov, Product of Design Mechatronics and Environment Department, \\ Brasov, Romania
}

\begin{abstract}
Insoles represent a mean by which humans can improve postural comfort or existence and manifestation of posture static and dynamic. The variety of materials and sizes for different kinds of foot insoles are for manufacturers a challenge in terms of getting products flexible, versatile and easily adaptable to a larger range of subjects. Biomechanical foot dysfunction influences plantar load distribution and mechanical tissue stress. Greater influence over plantar loads and tissue stress may lead to optimize the insole design to improve foot biomechanics. Some investigations founded that the use of cushioned insoles reduces the risk of stress fractures and injuries; other research has shown no protective effect. The use of cushioned or shock-absorbing insoles invoked reducing the impact forces associated with running, protecting against injuries. The paper emphasizes a new method for analysis of the insoles materials behavior in different alkalis and acids, similar to those encountered in real situations when walking and standing. The behavior study of composite structure insoles started to establish constructive variants and the modality of exposure of these samples at different degrees of softening in simple water, in water with salinity and under negative and positive temperature, also to mechanical stress.
\end{abstract}

\section{Introduction}

Insoles represent a multitude of means by which humans can improve comfort and postural dysfunctions existence and manifestation of a static posture and/or dynamic. The variety of materials and sizes as appropriate to different kinds of foot insoles are for manufacturers a challenge in terms of getting those products flexible, versatile and easily adaptable to a range of subjects as large [1].

Ensuring postural comfort through the use of well-made insoles and performance characteristics is the most important requirement in the construction category of footwear, either daily wear or for sports activities. As established in international standards there are a number of categories of shoes that protect human loco-motor system. "Safety shoes or boots with compression protection are required for work where a heavy object or item may roll over or a heavy animal may step on the toe (e.g., operating skid trucks and pallet jacks, working around bulk paper rolls or heavy pipes, etc.). In accordance with ASTM

${ }^{*}$ Corresponding author: braun@unitbv.ro 
performance standards - USA, compression resistant footwear must also meet impact resistance requirements. Again, there are two classes for compression resistance: class 50 which provides protection against exposure to a compressive force of $794 \mathrm{~kg}$ and class 75 which provides protection against exposure to a compressive force of $1134 \mathrm{~kg}$ " [2]. There are also other categories of standardized limits for planting surface protection of human loco-motor system, like: metatarsal protection, penetration of footwear bottoms, chainsaw use, electric shock, electric conduction, static dissipation, dielectric insulation, ankle protection, water resistant, chemical resistant, thermal insulated (cold).

Another important issue, with applications in rehabilitation of patients with diseases on the plantar surface is the choice of materials "when the physical and mechanical properties - durability, resilience, compressive stiffness, and coefficient of friction - are generally taken as the key requirements for the evaluation and selection of suitable fabrication materials for orthotic insoles" [3].

As shown by the author in his paper, there are still a number of "traditional tests which also neglect the importance of comfort perception in foot orthotic treatment, which affects the rate of compliance. A new in-shoe microclimate, which is relatively higher in temperature and humidity than the outer environment, is created during gait. Factors presented in previous experience, presence of pain or injury, neurophysiology and psychological issues, as well as the design, contour, and hardness of insole manufacturing may contribute to the overall comfort perception of foot orthoses". Many companies or clinics specialized in performance athletic footwear made a series of crash tests for soles with or without insoles to assess resistance to jumping, falling or shares with high energy loads. "Therefore, the energy absorbed ( $\Delta E$ between with and without insole conditions as having the same peak deceleration) by the insole could be calculated from the regression equations as follows:

$$
a_{s}=\alpha \times E+\beta=\alpha_{i} \times E_{i}+\beta_{i}
$$

where $\alpha, \beta, \alpha_{i}$, and $\beta_{i}$, are the coefficients of the linear regression equations for without insole and with insole conditions, respectively. The term $\mathrm{a}_{s}$ indicates the same peak deceleration under each condition, and $E$ and $E_{i}$ are the impact energies resulting in the same peak deceleration for without insole and with insole conditions, respectively; $E_{i}$ is larger than $E$ because of the cushioning ability of the insole, and:

$$
\Delta E=E_{i}-E=\left[\left(\alpha-\alpha_{i}\right) \times E_{i}+\left(\beta-\beta_{i}\right)\right] \alpha
$$

For another testing condition, the shock absorption foam was interposed below the insole. With the same way, the energy absorbed by the shock absorption foam could be calculated from the regression equations with insole only and with insole plus shock absorption foam conditions" [4].

Other researches on the insoles were focused on analyzing the methods of materials selection used to achieve them, focusing on their proper selection after simulation and modeling activities. As materials, producers have chosen those, based on polyethylene due to the configuration stability and possess adequate flexibility and strength. "Foot patterns are studied and tested to identify the different pressure at the insole to produce results in stress, displacement, and deformation" [5].

In most analysis of insoles behavior and after choosing materials, research has focused on determining the parameters of these mounting positions through their use in orthopedic studies on the forms of walking, running or jumping. Thus the concept of senzorimotor insoles was developed and built for applications in musculoskeletal rehabilitation. With this concept many patients could improve their gait without an operation of their musculoskeletal system [6]. 
"In an attempt to gain a greater understanding of the characteristics of orthotics materials and how they affect normal gait, Healy (2012) performed a repeated measures study analyzing kinematics and plantar pressures of 10 healthy participants while walking on a treadmill in various conditions, including shoes only, shoes with 4 types of flat insoles and 4 types of custom orthoses. The material properties for both custom and non-custom insert conditions included one of each of:

1. Low density polyurethane (PU) (Shore A hardness 20-25),

2. Medium density PU (Shore A hardness $55 \pm 3$ ),

3. Low density ethyl vinyl acetate (EVA) (Shore A hardness 25) and

4. Medium density EVA (Shore hardness 50) [7].

These materials were selected based that these were commonly prescribed in orthoses for individuals with diabetes. "Results for the flat insoles indicated that medium density EVA produced the greatest peak pressure. Low and medium density PU were most effective at increasing average contact area and reducing the pressure time interval. In the custom orthoses category there were no significant differences when compared to the shoe only condition, but medium density PU increased the average contact area by a greater percentage than the other materials. The authors concluded that medium density PU may have a different loading characteristic than the others, indicating it to be most suitable for patient with diminished plantar pressure sensitivities" [7].

\section{Method to evaluate the behavior of insoles}

The study consisted of two steps: the first step was related to analysis of water influence on insoles materials and the second step was related to analysis of cold and warm environment influences on the same insoles materials. First the first step of studies 7 samples of insoles was choosing (Fig.1.) to be analyzed in different environmental conditions (immersion in water and water solution, cold/warm environment and mechanical pressure).

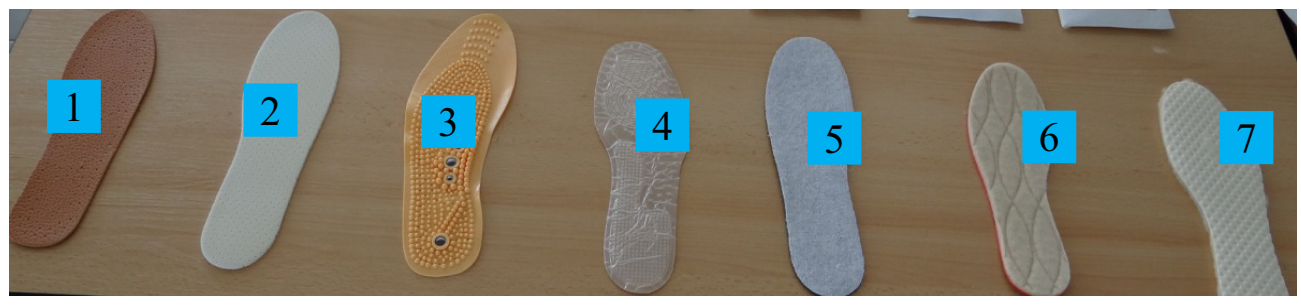

Fig. 1. The used materials for plantar shoe insole.

In the first step, two samples of each insole category were weighed, in dry conditions, at ambient temperature of $19^{\circ} \mathrm{C}$. After this step two samples of each insoles were then placed completely in water, in 5\% saline solution (two samples of each insoles), other two samples of each insoles insert into refrigerator at minus $18^{\circ} \mathrm{C}$ (during 12 hours), other two samples of each insoles placed in a heating chamber (dry autoclave) up at $50^{\circ} \mathrm{C}$ temperature (during 1 hour) and other two samples of each insoles placed under a mechanical force during 24 hours.

Entire duration of immersion in the prepared solutions was 7 days and weighing was performed after 1 hour, 2 hours, 24 hours, 48 hours and after 7 days for each sample. Each sample was monitored for 7 days from a visual standpoint and also the environmental parameters that were these samples (neutered containers, monitored temperature to remain constant throughout the experiment, natural and normal lighting - $200 \mathrm{Lx}$ ). 


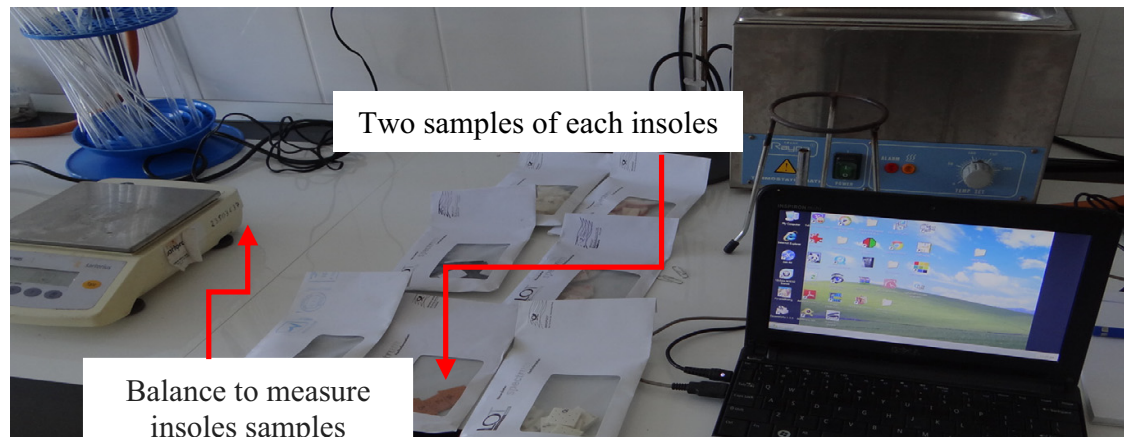

Fig. 2. The procedure of weighing the insoles samples before immersion in water, saline, dry autoclave and refrigerator and also after immersion process.

The duration of immersion was determined according to the determination of the samples estimated average exposure period after which to emphasize the substantial changes at the level of structure in the insoles construction.

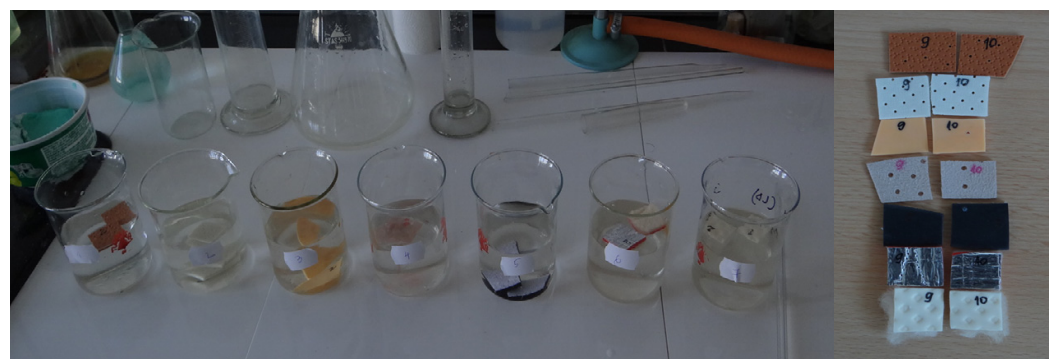

Fig. 3. The procedure of insoles immersion in water and saline.

\section{Results of experiments}

In the first phase of experiments was conducted to the weight's variation determination two samples for each type of insoles - immersed in water (Fig. 4.), and in saline (Fig. 5.), both at constant temperature of $21^{\circ} \mathrm{C}$.

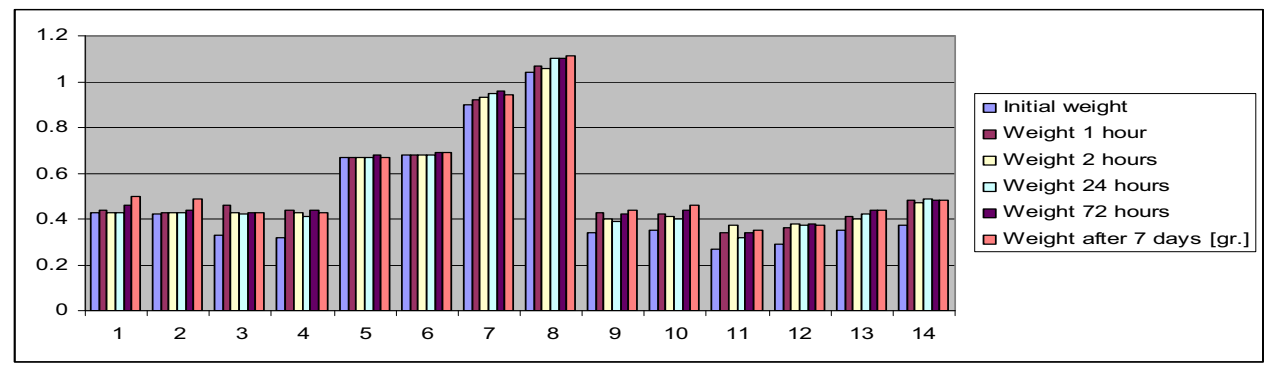

Fig. 4. The weight variation for insole samples immersed in simple water.

For samples immersed in saline, weight variation undergoes a series of changes from situation of immersion only in simple water which indicates a particular behavior of insoles samples. 


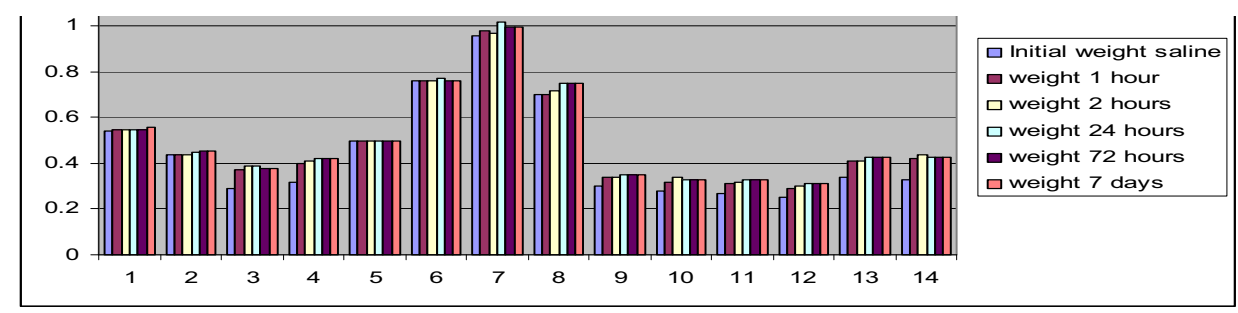

Fig. 5. The weight variation for insole samples immersed in saline.

However, there are a set of insoles that almost did not react at all when immersed in both water and saline, namely insoles samples made by silicon with magnetic pills. Also, procedures for sample introduction of insoles in refrigerator at a temperature of $-18^{\circ} \mathrm{C}$ and others under the action of mechanical forces did not lead to weight variation during the experiment. The only procedure that changed in less insoles weight was that they were placed in dry autoclave at temperature of $+50^{\circ} \mathrm{C}$. The weights of all samples decreased by an average of $2.5 \%$ which may be considered as not substantially alter their weight.

In a next step the samples were analyzed by a digital microscope, before and after immersion in water, saline, chilled and placed in dry autoclave. The images of the insoles were captured with a digital microscope type Keyence VHX-600 having magnifications between 20x and 5000x. Insoles material behaved differently especially the absorption of water and saline. In case of heat stress they have behavioral changes (stiffness/flexibility, brittleness/softening, color changed) immediately after removing from refrigerator and respective dry autoclave, they still returning to initial quality about 30 minutes.

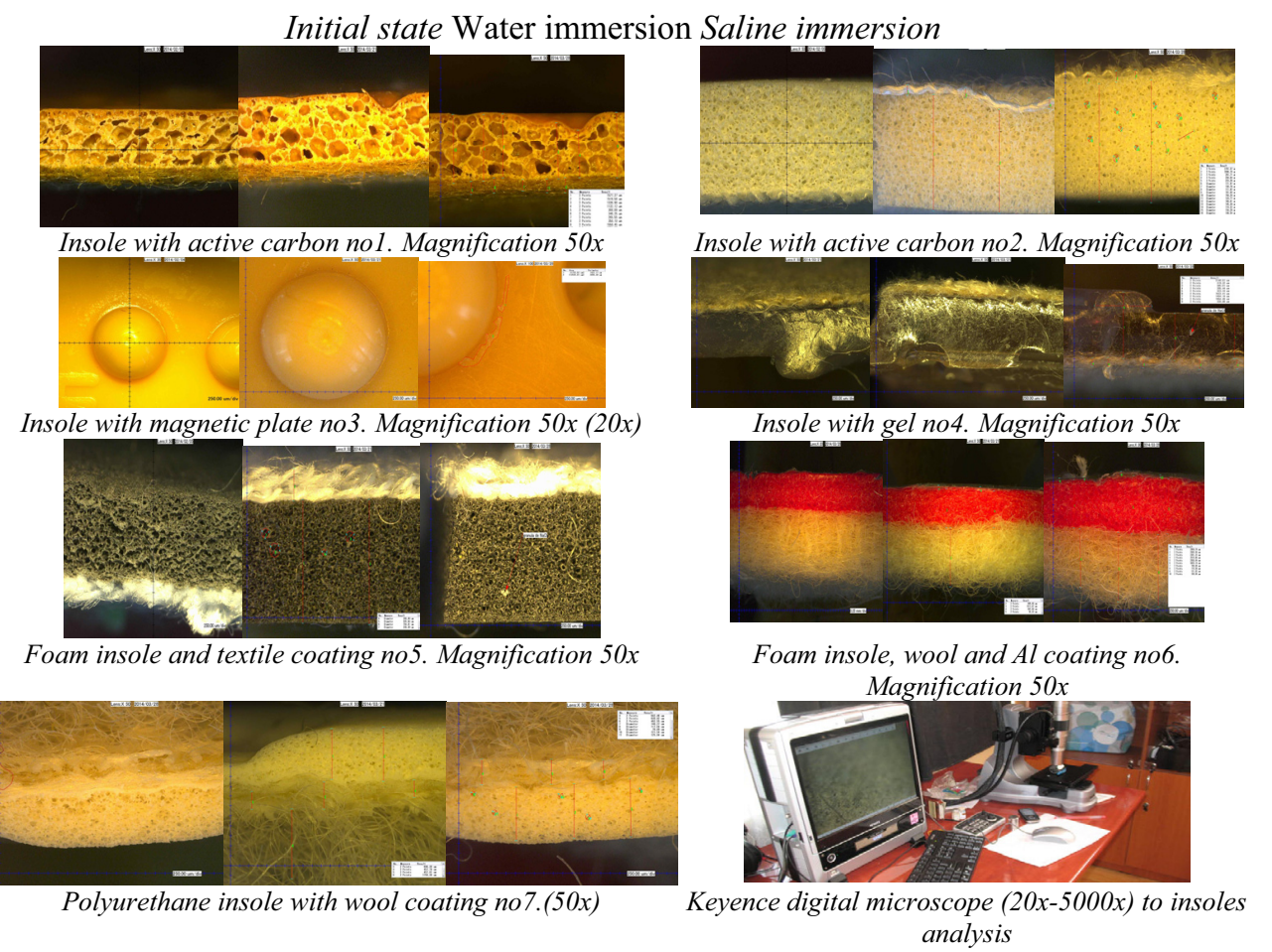

Fig. 6. Microscope images of insole samples in initial state, water immersion and saline immersion. 


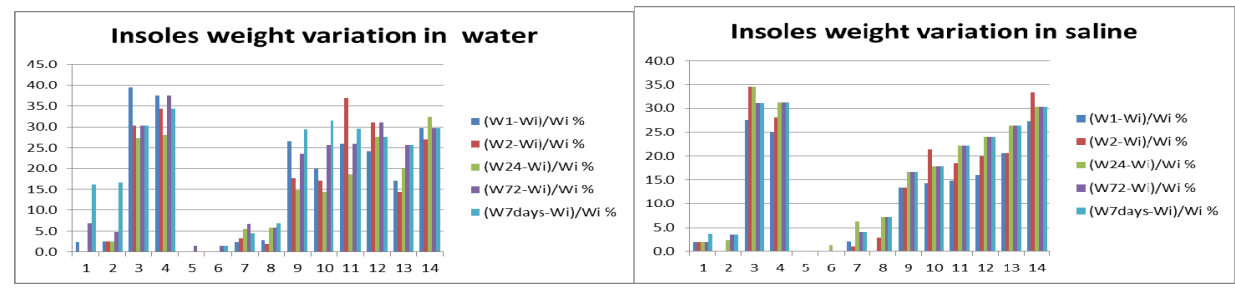

Fig. 7. Insoles weight variation in simple water (left) and saline (1\%) (right).

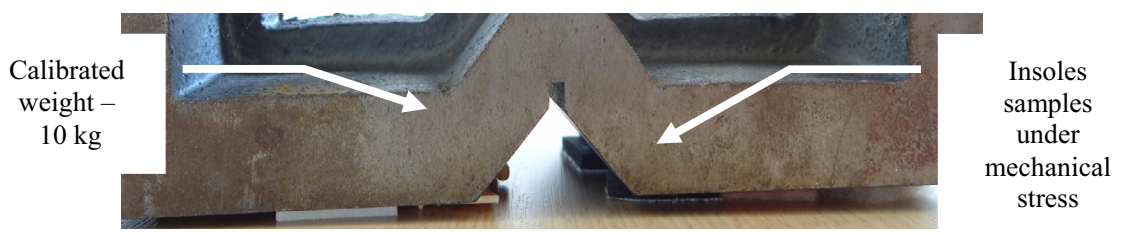

Fig. 8. Insoles samples under mechanical stress during 7 days.

In the case of insoles subjected to mechanical stress it has been observed a structure deformation, but returned to initial state in $30 \mathrm{~min}$. Insoles were under a weight equal to 10 $\mathrm{kg}$ for 7 days in the identical conditions to those that were analyzed in immersion $\left(21^{\circ} \mathrm{C}\right.$ ambient temperature, relative humidity $40 \%$, constant atmospheric pressure $750 \mathrm{~mm} \mathrm{Hg}$ ).

The size of these insoles samples evaluated before and after exposure to mechanical stress (assessments conducted with digital microscope) was their thickness in the pressure area with calibrated weight of $10 \mathrm{~kg}$. (Fig. 8.). Thus it is found that the most important structural changes of the analyzed insoles material samples were occurred at the stage of immersion in water and saline.

\section{Conclusions}

After analyzing the data obtained from measuring the weight of insoles sample we can say that these materials react most to the absorption of liquid - water, and forms a compact structure especially those that are made of foam and polyurethane. In case of foam insoles samples, polyurethane or those reached with active carbon, immersed in saline, the salt crystals enter in spaces of structure and when water evaporates, the crystals change the structure stiffen of insoles.

Correlating the actions of stress on the effects on insoles material structure we're able to identify the following aspects: Insoles made from silicone and gel-like material enriched with active carbon or magnetic tablets (insoles no.1, 3 and 4) partial change their color, do not absorb water or saline, not brittle after exposure to low or high temperatures and the main dimension (thickness) does not change after exposure to mechanical stress; Foam or polyurethane insoles (insoles no. 2, 5, 6 and 7) absorb much more quantity of water or saline and contact materials (wool, fabric protective, Al foil) with foot or the shoe surface undergoes changes of appearance, color or even come off from the insole base.

Therefore the behavior analysis of insoles selected types highlighted the importance of keeping the initial characteristics (flexibility, constant size, elasticity and toughness) to give comfort and ease of use on the plantar surface of the human foot. Any physical or chemical aggression on these insoles structures, their use in inappropriate conditions may cause discomfort, dysfunction in the gait cycle or may develop over time even local damages.

The next step in our research will mean to capture, to process and to interpret the images in terms of longitudinal and transversal sections to the tested insoles, due to the 
used digital microscope, having the possibility to measure the degree of material deformation under mechanical, thermal and chemical conditions. In this way it will be possible to obtain a statistical conclusion related to all categories of foot insoles behavior, for all condition.

This paper is part of the current researches in Applied Optometric Laboratory and Advanced Mechatronic Researches Center from University Transilvania Brasov.

\section{References}

1. M. Baritz et al., Microscopic analyze of insoles composite structures behavior under water immersion, temperature variation and saline, Conference POLCOM Bucharest (2014)

2. University of Nebraska Lincoln-Environmental Health and Safety, Personal Protective Equipment (PPE) - Foot Protection Evaluation And Checklist, http://ehs.unl.edu (2015)

3. W. L Ting et al., JRRD 51, 311-324 (2014)

4. H. Chiu et al., J. of Appl. Biomech. 23, 119-127 (2007)

5. J. Whaley et al., EURECA, 41 - 42, (2013)

6. S. Kolling et al., Material modeling of orthopedic insoles, $7^{\text {th }}$ European LS-DYNA Conf. (2009)

7. Pedorthics Quatrterly, Pedorthic Association of Canada (2012) 